\title{
Acercamiento al emprendimiento responsable a través de experiencias reales
}

Cristina López-Cózar Navarro ${ }^{a}$, Tiziana Priede Bergamini ${ }^{b}$ y Sonia Benito Hernández

a Profesora de la Universidad Politécnica de Madrid cristina.lopezcozar@upm.es, ${ }^{b}$ Profesora de la Universidad Europea de Madrid tiziana.priede@universidadeuropea.es y ${ }^{\mathrm{c}}$ Profesora de la Universidad Politécnica de Madrid sonia.benito@upm.es.

\begin{abstract}
Entrepreneurial spirit, ethical commitment and social responsibility at work are today among the most demanded competencies by society. This paper presents an initiative that aims to contribute to the promotion of a sustainable and inclusive entrepreneurship. With this purpose, a semminar with social entrepreneurs was organized. After presenting their own experiences the social entrepreneurs, acted as consultants; in this way, they offered advice for the student in the the development of the business plans required at the end of the subject; solving their doubts and proposing improvement actions for their business ideas.
\end{abstract}

Keywords: social entrepreneurship, engagement, responsible behavior, skills, learning, higher education.

\footnotetext{
Resumen

Entre las competencias más demandadas por la sociedad en el momento actual destacan tanto el espíritu emprendedor como el compromiso ético y la responsabilidad social en el trabajo. En el presente trabajo se presenta una iniciativa con la que se pretende contribuir a la promoción de un emprendimiento sostenible e integrador. Con esta finalidad se organizó una jornada con emprendedores sociales quienes, tras exponer sus propias experiencias, actuaron como consultores. De esta manera, ofrecieron asesoramiento al alumnado para la elaboración de los planes de negocio que deben realizar al final de la materia, resolviendo sus dudas y proponiéndoles acciones de mejora para sus ideas de negocio.
}

Palabras clave: emprendimiento social, compromiso, comportamiento responsable, competencias, aprendizaje, educación superior.

\section{Introducción}

Tras el largo periodo de crisis económica vivida en nuestro país, en la actualidad se debaten nuevos modelos de desarrollo con el fin de lograr, no solo la recuperación, sino también que 
en el futuro, se alcance un crecimiento sostenible e integrador. En este contexto, nos parece relevante que los jóvenes universitarios adquieran conocimientos y destrezas relacionadas precisamente con el objetivo de crear empresas que sean capaces de ser rentables $\mathrm{y}$, al mismo tiempo, responsables, de manera que generen valor para la comunidad en su conjunto.

Efectivamente, una de las competencias más demandadas por la sociedad a los egresados universitarios en la actualidad es el espíritu emprendedor. Siguiendo a Bacigalupo et al. (2016) se considera una competencia trasversal clave, tanto individual como grupal, y se define como la capacidad de reconocer oportunidades y actuar sobre ellas para lograr transformar ideas en valor para otros, ya sea valor financiero, cultural o social (para una revisión de la literatura sobre la definición de esta competencia desde las diferentes perspectivas ver: Marina, 2009). Desde la Unión Europea se señala la necesidad de educar en el fomento del espíritu emprendedor, especialmente en las universidades, y en este sentido se han puesto en marcha numerosas iniciativas dentro de la política comunitaria (Sobrado y Fernández, 2010). Lo cual implica que es preciso desarrollar en los estudiantes universitarios determinadas cualidades y habilidades educables e imprescindibles, necesarias para formar personas con empuje y resolución ante el entorno que les rodea.

No obstante, el emprendimiento debe estar sustentado sobre la base de un comportamiento responsable y un compromiso ético en el trabajo. De hecho, entre las ocho competencias que la Comisión Europea considera clave para el aprendizaje permanente, se encuentran el sentido de la iniciativa y espíritu de empresa, así como competencias sociales y cívicas. Igualmente, en la recomendación 2006/962/CE, se señala expresamente la necesidad de tomar conciencia sobre los valores éticos e impulsar actividades de apoyo a la cohesión social y al desarrollo sostenible (COM, 2006).

En esta línea, tanto desde las administraciones públicas (COM, 2013), como diferentes autores (Juliá, 2013; Melián Navarro et al., 2017; Priede Bergamini et al., 2017; Hernández et al., 2018; Sánchez Espada et al., 2018) abogan por que la actitud emprendedora se desarrolle dentro del ámbito de la empresa social.

El emprendimiento social se presenta como una alternativa de negocio diferente y especial, capaz de identificar una oportunidad en la resolución de un problema social o ambiental. Se trata, pues, de personas que deciden montar una empresa con la intención de llevar a cabo una innovación social (Mulgan, 2006; Brown y Wyatt, 2010). La empresa social participa en la economía de mercado, pero, al mismo tiempo, aporta respuestas innovadoras y eficientes para ayudar a solventar necesidades de la sociedad no atendidas. Este tipo de proyectos empresariales persiguen la creación de valor social por encima del ánimo de lucro (Martin y Osberg, 2007; Defourny y Nyssens, 2012; Priede Bergamini et al., 2014; López-Cózar Navarro et al., 2015). Así pues, en el presente trabajo se presenta una iniciativa con la que se pretende contribuir a la promoción de un emprendimiento sostenible e integrador enfocado desde la experiencia real. 


\section{Objetivos}

Hoy más que nunca, desde las aulas universitarias es preciso formar a profesionales y líderes empresariales responsables y comprometidos, con el propósito de conseguir empresas económicamente viables, pero también sostenibles y que estén orientadas hacia el fortalecimiento de un orden socioeconómico más justo y equilibrado.

Por lo tanto, el objetivo del presente trabajo es dar a conocer una actividad docente orientada a la formación en competencias llevada a cabo con estudiantes de postgrado de la Universidad Politécnica de Madrid durante el segundo semestre del curso 2018-2019. Con el propósito de fomentar el desarrollo de iniciativas empresariales ligadas a proyectos responsables, sostenibles e integradores, se organizó una jornada sobre emprendimiento social. Para ello se contó con la colaboración de tres emprendedores sociales quienes, además de compartir su experiencia personal, se involucraron en el proceso de aprendizaje de los estudiantes. Consideramos que se trata de una actividad interesante para la comunidad académica, dado que se puede adaptar fácilmente para realizarla en asignaturas relacionadas con el área de Organización de empresas de cualquier titulación universitaria.

\section{Desarrollo de la innovación}

En este epígrafe se presenta el contexto en el que se ha llevado a cabo la experiencia y se describe la misma.

\subsection{Contextualización}

La experiencia presentada se ha desarrollado en la asignatura Creación de empresas. Se trata de una asignatura optativa, de 4 ECTS, que se imparte de manera conjunta en el segundo semestre del Máster Universitario en Economía Agraria, Alimentaria y de los Recursos Naturales y en el cuarto semestre del Máster Universitario en Ingeniería Agronómica, de la Escuela Técnica Superior de Ingeniería Agronómica, Alimentaria y de Biosistemas de la Universidad Politécnica de Madrid.

En la primera clase del curso, se conciencia al alumnado sobre la trascendencia de la creación de nuevas empresas para el crecimiento económico de cualquier país; se presenta el emprendimiento como una actividad fundamental para la generación de empleo, el desarrollo de innovaciones y la mejora de la productividad, logrando que la economía sea más competitiva. Así mismo, se incide sobre la importancia de definir adecuadamente la actividad del nuevo proyecto empresarial y de realizar una correcta planificación de la puesta en marcha.

A continuación, durante las siguientes semanas, se imparten una serie de clases teóricas donde se explican los temas relacionados con los principales aspectos que tiene que contener un plan de negocio: descripción de la actividad de la empresa, el producto o el servicio que se va a ofrecer; identificación y análisis del mercado; plan de marketing; localización, costes, producción y operaciones; organización y recursos humanos; financiación y análisis 
económico-financiero; aspectos formales y jurídicos del proyecto, etc. Estas primeras sesiones teóricas son importantes para sentar las bases que les permitan poder desarrollar posteriormente el trabajo práctico, consistente precisamente en la elaboración de un plan de empresa.

Durante el resto del curso, los alumnos tienen que realizar el citado trabajo con el que se pretende conseguir que adquieran los conocimientos y las habilidades necesarias para enfrentarse al reto de crear una empresa. Para ello, se forman grupos compuestos por 2 o 3 personas y se les explica que al final tienen que entregar un trabajo escrito y realizar dos presentaciones orales. En la primera de ellas, los diferentes equipos tienen que exponer su idea de negocio, especificando qué necesidad pretenden satisfacer y a qué público se quieren dirigir; ya que la definición del producto y el mercado es previo al desarrollo de las demás partes del plan de empresa y se considera un aspecto crucial del mismo. La segunda presentación se realiza a final de curso y en ella deben exponer las principales partes del trabajo desarrollado y las conclusiones más relevantes derivadas del proceso de creación. La información detallada con el contenido completo del plan de empresa quedará recogida en un documento escrito que deberán entregar al finalizar el curso.

\subsection{Descripción de la experiencia}

Previo a la primera exposición oral (presentación de las ideas de negocio), se propuso realizar un encuentro con emprendedores sociales con la finalidad de que el alumnado tuviera la oportunidad de acercarse a esta realidad empresarial, desconocida por la mayoría de los estudiantes. La actividad se planteó con un doble objetivo: por un lado, se pretendía despertar entre los estudiantes el interés por el emprendimiento social mediante el contacto directo con iniciativas reales. Por otro lado, se planteó como una oportunidad para que los alumnos pudieran validar la idoneidad de sus proyectos empresariales.

Así pues, la jornada se organizó en dos partes; en primer lugar, se presentó una mesa redonda con tres emprendedores sociales invitados, los cuales presentaron de forma breve y atractiva sus respectivas experiencias explicando claramente qué es el emprendimiento social, cómo surgió la idea de negocio a raíz de la resolución de un problema social, las principales dificultades encontradas, así como sus principales satisfacciones y logros.

Los tres casos presentados son empresas sociales que cuentan con una trayectoria en el tiempo que garantiza tanto la viabilidad económica financiera de la iniciativa, como el impacto social alcanzado en su comunidad. En particular, contamos con la presencia de una emprendedora social que con su proyecto combate la trata de personas; con el socio fundador de una empresa que ofrece productos ecológicos para agricultura y jardinería, con los que pretende lograr que dichas actividades se realicen de manera sostenible; y con el cofundador de una empresa destinada a personalizar el aprendizaje y facilitar la integración en el mercado laboral de los estudiantes.

En la segunda parte de la actividad, fueron los propios estudiantes quienes intervinieron, exponiendo ante los tres emprendedores sus posibles ideas de negocio, las principales dudas y los problemas que encontraban a la hora de plantear sus proyectos de empresa. Los 
emprendedores invitados actuaron como consultores, escuchando al alumnado y ofreciendo opiniones, apoyo y asesoramiento. De esta manera, los diferentes equipos de estudiantes fueron planteando las dificultades y amenazas detectadas, y los emprendedores se involucraron en la resolución de las mismas; aportando su experiencia y su conocimiento, les hicieron observaciones y recomendaciones, y les propusieron soluciones así como diversas alternativas.

\section{Resultados}

Con el propósito de conocer la utilidad de la experiencia y el grado de satisfacción con la misma, se realizó una encuesta a los estudiantes. Así, una vez finalizada la jornada, se les plantearon una serie de cuestiones, asegurando el anonimato de las respuestas. Para ello, se elaboró un cuestionario con 10 ítems a valorar sobre una escala Likert de 1 (muy bajo) a 5 (muy alto), y tres preguntas abiertas, en las que se pedía señalar los aspectos más positivos y negativos de la jornada. En la tabla 1 se exponen dichas variables.

Tabla 1. Preguntas planteadas en la encuesta de satisfacción

Utilidad de la jornada en tu proceso de aprendizaje

Aumenta el nivel de aprendizaje sobre la empresa social

Incrementa el nivel de aprendizaje sobre la asignatura

Acercamiento a la realidad empresarial

Despierta el interés por la responsabilidad social y prácticas empresariales responsables

Presenta alternativas nuevas para tu fututo profesional

Despierta tu compromiso propio como ciudadano

Después de esta jornada, te planteas el emprendimiento como una opción laboral inmediata

Después de esta jornada, te planteas el emprendimiento como una opción laboral a medio plazo

Valoración global de la jornada

Fuente: Elaboración propia

La figura 1 se muestran los resultados de la encuesta, con la valoración de los estudiantes según su nivel de satisfacción con cada ítem. 


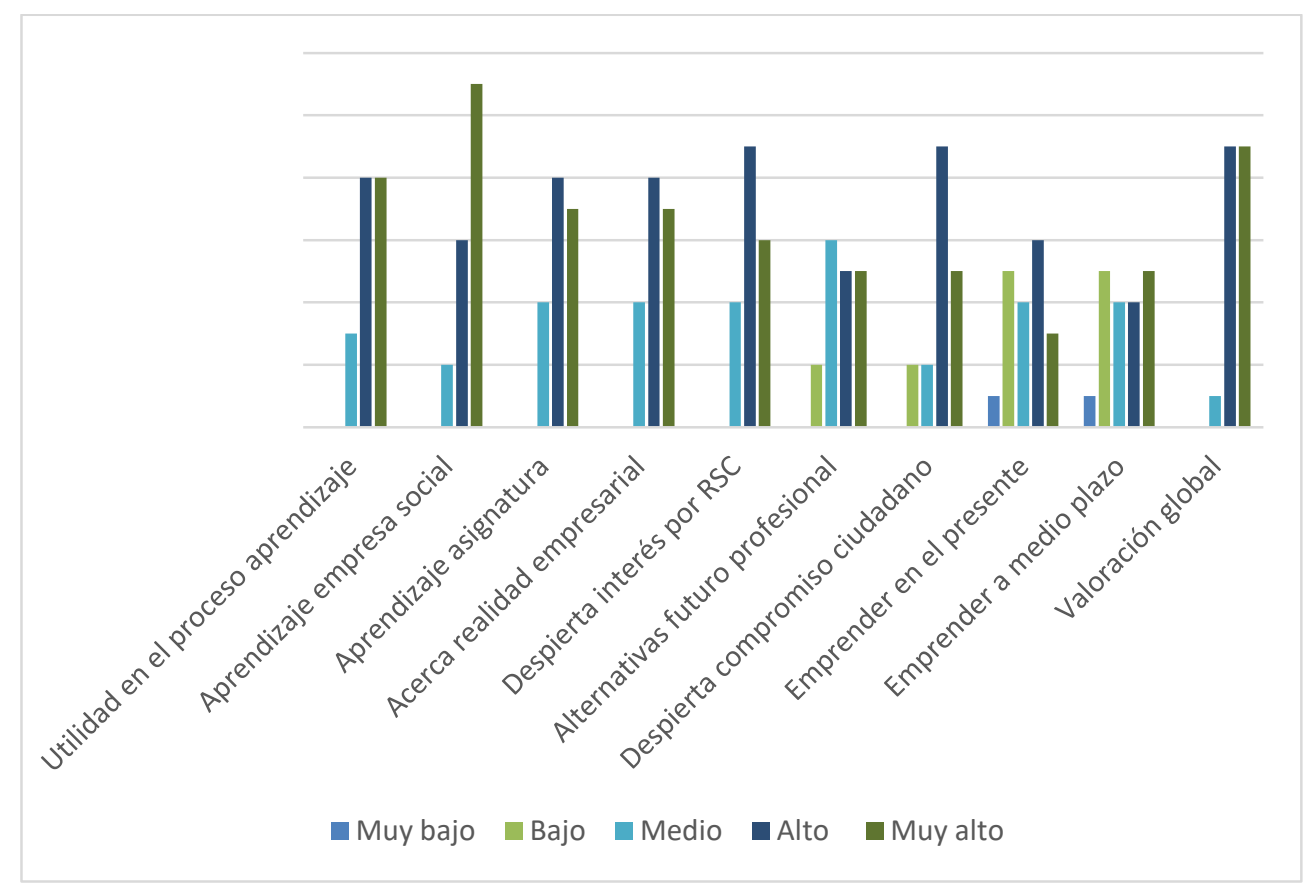

Fig. 1 Resultados encuesta de satisfacción

Los resultados de la encuesta realizada muestran que el grado de satisfacción de los alumnos con la experiencia es muy elevado. Ante la pregunta "Utilidad de la jornada en tu proceso de aprendizaje", el 84\% de las respuestas obtenidas se sitúa en las categorías alto o muy alto. Un $79 \%$ de estudiantes considera que ha incrementado su nivel de aprendizaje sobre el contenido de la asignatura en general, y el $89 \%$ considera que ha aumentado su nivel de aprendizaje, en particular, sobre la empresa social. Asimismo, cabe destacar la respuesta obtenida a las preguntas sobre la responsabilidad social empresarial y el compromiso personal como ciudadano; en ambos casos, el porcentaje de respuestas en las categorías alto o muy alto se sitúa por encima del 70\%. En concreto, 14 de los 19 estudiantes afirman que se ha despertado su compromiso como ciudadanos, y 15 consideran que se ha incrementado su interés por las prácticas responsables en las empresas.

Respecto a las preguntas sobre la intención de emprender un nuevo proyecto empresarial en el futuro, la reacción del alumnado fue menos alentadora de lo esperado. En los tres casos, las respuestas obtenidas son mucho más dispersas que en el resto de las cuestiones planteadas. En particular, el 52\% considera que el emprendimiento social puede ser una alternativa interesante para su futuro profesional, pero la mayoría no tiene intención de emprender un nuevo proyecto ni en el corto, ni en el medio plazo. Por último, la respuesta a la pregunta "Valoración global de la jornada" se considera muy satisfactoria pues la inmensa mayoría de estudiantes (18 de los 19), se posicionan en las dos categorías superiores.

Por su parte, la encuesta también incluía tres preguntas abiertas en las que se pedía a los estudiantes que manifestaran su opinión sobre los aspectos más positivos y más negativos de 
la jornada, así como posibles sugerencias o mejoras para el futuro. Los alumnos, en general, inciden nuevamente en su valoración favorable; destacaron el acercamiento a la empresa social y señalaron que les resultó de gran interés las experiencias narradas por los tres emprendedores. Así mismo, la mayoría también destacó el valor de los comentarios y consejos recibidos. Por lo que se refiere a las recomendaciones para el futuro, varios estudiantes señalaron que debería repetirse en los próximos cursos.

\section{Conclusiones}

Este trabajo ha presentado el desarrollo de una actividad formativa de acercamiento al emprendimiento social y a las prácticas empresariales responsables desde la realidad. Esta actividad ha pretendido, por una parte, desarrollar el espíritu emprendedor ya que se circunscribe a una asignatura de creación de empresas; pero por otra, ha buscado también el carácter responsable, el comportamiento ético en el trabajo y la posibilidad de generar un impacto positivo en la comunidad. Aspectos todos ellos muy relevantes para nuestro contexto económico actual. Consideramos, pues, esta actividad muy oportuna y precisamente enfocada a estudiantes de posgrado, con un nivel de madurez elevado y en un momento idóneo para desarrollar competencias en este sentido y orientarse hacia un emprendimiento responsable en su área de conocimiento.

El análisis de los resultados de la encuesta realizada permite concluir que la mayoría de los estudiantes implicados considera que se trata de una actividad formativa útil. Según ellos mismos han manifestado, promueve el aprendizaje sobre la asignatura en general y, en particular, sobre el emprendimiento social. Igualmente, los resultados muestran que ha conseguido despertar su interés por la responsabilidad social empresarial y su propio compromiso como ciudadano. No obstante, hay que señalar que también se ha detectado que la intención de emprender genera cierta reticencia e incertidumbre, dadas las dificultades y barreras con las que se encuentran los jóvenes emprendedores a la hora de poner en marcha sus ideas; es posible que los estudiantes de posgrado sean más conscientes de las dificultades dado su mayor nivel de conocimiento y madurez, y ello haya provocado respuestas más dispersas en este sentido.

Los tres casos expuestos: trata de personas, productos ecológicos para lograr una agricultura y jardinería sostenible e integración de jóvenes en el mercado laboral, son representativos de la amplia variedad campos que puede abarcar el emprendimiento social. Como puede observarse, se trata de empresas de distintos sectores y que tratan de dar respuesta a problemas sociales y medioambientales muy diferentes. Estos ejemplos de emprendimiento social permitieron a los alumnos conocer sus diversidades en profundidad y aprender de las experiencias reales. Así pues, desde la perspectiva docente, se ha alcanzado el objetivo de ofrecer alternativas profesionales al alumnado encaminadas a la consecución de un futuro más justo y equilibrado.

Efectivamente, es importante resaltar la idoneidad de desarrollar este tipo de iniciativas que promueven un aprendizaje integral del alumnado dado que están en coherencia con el modelo de formación que debemos perseguir en el EEES. En este sentido, resulta fundamental que 
el estudiante se vea expuesto a actividades docentes que incluyan, simultáneamente, distintos elementos de su formación, tanto en lo que se refiere a conocimientos teóricos, como en lo referente a objetivos competenciales más complejos. El contacto directo con fundadores de empresas y la posibilidad de debatir con ellos diferentes aspectos de sus propias ideas de negocio, permite consolidar los conocimientos empresariales y fomenta el espíritu emprendedor de los estudiantes, así como otras competencias educables inherentes al proceso de creación tales como la creatividad, la responsabilidad, la perseverancia, la asunción de riesgos o la capacidad de decisión, etc.; las cuales determinarán el comportamiento de los futuros egresados en su vida profesional.

En definitiva, se puede decir que, pese a la dificultad que puede suponer la organización de actividades como la descrita, pues requiere un considerable esfuerzo de coordinación de todos los implicados, merece la pena poner en práctica iniciativas de este tipo, ya que, como se ha puesto de manifiesto, repercute de forma muy positiva en el proceso de aprendizaje del alumnado. Por su parte, se concluye también que los estudiantes pueden prepararse para ser empresarios y creadores de empleo, ya que la formación y la experiencia son variables muy relevenates en este sentido; desde la Universidad debe pues fomentarse la oferta de asignaturas enfocadas al desarrollo del emprendiemiento, así como la necesaria colaboración entre la universidad y la empresa.

\section{Referencias}

BACIGALUPO, M., KAMPYLIS, P., PUNIE, Y., y VAN DEN BRANDE, G. (2016). EntreComp: The entrepreneurship competence framework. Luxembourg, Publication Office of the European Union.

BROWN, T. y WYATT, J. (2010). “Design thinking for social innovation”. Stanford Social Innovation Review, winter, p. 30-35.

COMISIÓN EUROPEA (2006). Recomendación (2006/962/CE) del Parlamento Europeo y del Consejo de 18 de diciembre de 2006 sobre las competencias clave para el aprendizaje permanente. Diario Oficial L 394 de 30.12.2006.

COMISIÓN EUROPEA (2013). Comunicación de la Comisión al Parlamento Europeo, al Consejo, al Comité Económico y Social Europeo y al Comité de las Regiones. Plan de acción sobre emprendimiento 2020. Relanzar el espíritu emprendedor en Europa. Bruselas, 9.1.2013 COM (2012) 795 final.

DEFOURNY, J. y NYSSENS, M. (2012). «El enfoque EMES de la empresa social desde una perspectiva comparada”. CIRIEC-España, Revista de Economía Pública, Social y Cooperativa, vol. 75, pp. 7-34.

JULIÁ, J. F. (2013). "Tribuna de opinión: Emprendimiento y universidad. Una referencia al caso de España y a la UPV”. REVESCO. Revista de Estudios Cooperativos, vol. 113, p. 7-27.

HERNÁNDEZ, I., PÉREZ, C., y RUA, S. (2018). Intereses y perspectivas formativas en economía social y solidaria de los estudiantes universitarios. CIRIEC-España, Revista de economía pública, social y cooperativa, (94), 91-121. 
LÓPEZ-CÓZAR NAVARRO, C., PRIEDE BERGAMINI, T. y RODRÍGUEZ LÓPEZ, Á. (2015). Avaliación do marco legal para o emprendemento social en América e Europa. Revista Galega de Economía, vol. 24, issue 1, p. 101-110.

MARINA, J. A. (2010). "La competencia de emprender”. Revista de Educación, vol. 351, p. 49-71.

MARTIN, R. y OSBERG, S. (2007). “Social entrepreneurship: The case for definition”. Stanford Social Innovation Review, vol. 5, issue 2, p. 28-39.

MELIÁN NAVARRO, A., CAMPOS I CLIMENT, V., y SANCHIS PALACIO, J. R. (2017). "La educación de postgrado en Economía Social en la universidad española ¿una asignatura pendiente?” CIRIEC-España, Revista de Economía Pública, Social y Cooperativa, Vol. 89, p. 33-54.

MULGAN, G. (2006). “The process of social innovation”. Innovations: Technology, Governance, Globalization, Vol. 1, issue 2, p. 145-162.

PRIEDE BERGAMINI, T., LÓPEZ-CÓZAR NAVARRO, C. y RODRÍGUEZ LÓPEZ, A. (2014). "Análisis del marco económico-jurídico específico para los emprendedores sociales. Un estudio comparado entre diversos países". CIRIEC-España Revista de Economía Pública, Social y Cooperativa, vol. 80, p. 115-138.

PRIEDE BERGAMINI, T., LÓPEZ-CÓZAR NAVARRO, C., y BENITO HERNANDEZ, S. (2017). "Desarrollo competencial del alumno: el espíritu emprendedor y la responsabilidad social". En In-Red Congreso Nacional de innovación educativa y de docencia en red, Valencia: Editorial Universitat Politècnica de València. 918-928.

SÁNCHEZ ESPADA, J., MARÍN LÓPEZ, S., BEL DURÁN, P. y LEJARRIAGA PÉREZ DE LAS VACAS, G. (2018). "Educación y formación en emprendimiento social: características y creación de valor social sostenible en proyectos de emprendimiento social". REVESCO: Revista de Estudios Cooperativos, Vol. 129, p. 16-38.

SOBRADO FERNÁNDEZ, L., y FERNÁNDEZ REY, E. (2010). “Competencias emprendedoras y desarrollo del espíritu empresarial en los centros educativos”. Educación XX1, Vol. 13, issue 1, p. 1538. 